\title{
Entropy analysis of the EEG background activity in Alzheimer's disease patients
}

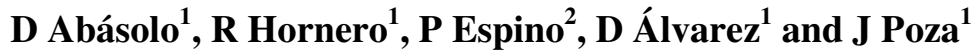 \\ ${ }^{1}$ E.T.S. Ingenieros de Telecomunicación, University of Valladolid, Camino del \\ Cementerio s/n, 47011, Valladolid (Spain) \\ ${ }^{2}$ Hospital Clínico San Carlos, c/Profesor Martín Lagos s/n, 28040, Madrid (Spain) \\ E-mail: danaba@tel.uva.es
}

\begin{abstract}
Alzheimer's disease (AD) is the most common neurodegenerative disorder. Although a definite diagnosis is only possible by necropsy, a differential diagnosis with other types of dementia and with major depression should be attempted. The aim of this study was to analyse the EEG background activity of $\mathrm{AD}$ patients to test the hypothesis that the regularity of the AD patients' $\mathrm{EEG}$ is higher than that of age-matched controls. We recorded the EEG from 19 scalp electrodes in 11 AD patients and 11 age-matched controls. Two different methods were used to estimate the regularity of the EEG background activity: Spectral entropy (SpecEn) and Sample entropy (SampEn). We did not find significant differences between AD patients and control subjects' EEGs with SpecEn. On the other hand, AD patients had significantly lower SampEn values than control subjects $(p<0.01)$ at electrodes P3, P4, O1 and O2. Our results show an increase of EEG regularity in AD patients. These findings suggest that non-linear analysis of the EEG with SampEn could yield essential information and may contribute to increase the insight into brain dysfunction in $\mathrm{AD}$ in ways which are not possible with more classical and conventional statistical methods.
\end{abstract}

Keywords: Alzheimer's disease, electroencephalogram, sample entropy, spectral entropy

PACS number(s): 05.45.Tp, 87.19.La, 87.80.Tq

\section{Introduction}

Alzheimer's disease (AD) is the most frequent cause of dementia, and is characterized by progressive impairments in cognition and memory whose course lasts several years prior to death (Jeong 2004). These clinical features are accompanied by characteristic histological changes in the brain, which include widespread cortical atrophy, intracellular deposition of neurofibrillary tangles, and extracellular deposition of senile plaques, particularly in the hippocampus and the cerebral cortex (Selkoe 1994). AD is considered to be the main cause of dementia in western countries (Bird 2001), and although a definite diagnosis is only possible by necropsy, a differential diagnosis with other types of dementia and with major depression should be attempted. Magnetic resonance imaging and computerized tomography can be normal in the early stages of $\mathrm{AD}$, but a diffuse cortical atrophy is the main sign in brain scans. Mental status tests are also useful. 
The electroencephalogram (EEG) has been used as a tool for diagnosing dementias for several decades. The hallmark of EEG abnormalities in AD patients is a shift of the power spectrum to lower frequencies and a decrease of coherence among cortical areas (Jeong 2004), although in the early stages of the disease the EEG may exhibit normal frequencies (Markand 1990). These abnormalities are thought to be associated with functional disconnections among cortical areas resulting from death of cortical neurons, axonal pathology, cholinergic deficits, etc. (Jeong 2004).

Recent progress in the theory of non-linear dynamics has provided new methods for the study of the EEG (Jeong 2004). Non-linearity as a necessary condition for chaotic behaviour is present in many dynamical systems found in nature, including the brain. Non-linearity in the brain is introduced even at the cellular level, since the dynamical behaviour of individual neurons is governed by threshold and saturation phenomena. Moreover, the hypothesis of an entirely stochastic brain can be rejected due to its ability to perform sophisticated cognitive tasks. Given the highly non-linear nature of the neuronal interactions at multiple levels of spatial scales, the EEG appears to be an appropriate area for non-linear time series analysis (Kantz and Schreiber 1997).

There are many studies in which non-linear time series analysis techniques were applied to different kinds of EEGs. These investigations of the electrical activity of the brain have revealed possible medical applications, since analysis based on non-linear dynamics yields information unavailable from traditional EEG spectral-band analysis (Pritchard et al 1994). Moreover, they have given rise to the possibility that the underlying mechanisms of the brain function may be explained by non-linear dynamics (Babloyantz and Destexhe 1988, Röschke et al 1995, Stam et al 1995). Particularly, several studies have examined the non-linear dynamics of the EEG in AD. It has been found that AD patients have lower correlation dimension $\left(D_{2}\right)$ values - a measure of dimensional complexity of the underlying system (Grassberger and Procaccia 1983a) - than control subjects (Pritchard et al 1994, Stam et al 1995, Jeong et al 1998, 2001a). These results show a decrease in the complexity of the electrical activity in brains injured by AD (Jeong 2004). The first Lyapunov exponent ( $L 1)$ has also been used to characterize non-linear behaviour (Wolf et al 1985). It has been shown that AD patients have significantly lower $L 1$ values than controls in almost all EEG channels (Jeong et al 1998, 2001a). However, the amount of data required for meaningful results in the computation of $D_{2}$ and $L 1$ is beyond the experimental possibilities for physiological data (Eckmann and Ruelle 1992). Moreover, the Grassberger and Procaccia algorithm or its modifications used to estimate the $D_{2}$ assume the time series to be stationary (Grassberger and Procaccia 1983b), something generally not true with biological data. Therefore, it becomes necessary to study the EEG background activity with different methods. For instance, mutual information analysis (Jeong et al 2001b) and synchronization likelihood (Stam et al 2003, Pijnenburg et al 2004) have been used to assess information transmission between different cortical areas in AD.

One possible alternative solution lies in computing the entropy of the EEG. Entropy is a concept addressing randomness and predictability, with greater entropy often associated with more randomness and less system order. Recently, a number of different estimators have been introduced to quantify the entropy of time series. These approaches may be loosely classified into two groups: spectral entropies and embedding entropies (Sleigh et al 2004). Spectral entropies estimate the changes in the amplitude component of the power spectrum of the EEG, using the amplitude components at each frequency of the power spectrum as the probabilities in the entropy calculations (Sleigh et al 2004). On the other hand, embedding entropies provide information about how the EEG signal fluctuates with time by comparing the time series with a delayed version of itself (Sleigh et al 2004).

The present study was undertaken to examine the EEG background activity in AD with two different entropy definitions: Spectral Entropy (SpecEn) and Sample Entropy (SampEn). SpecEn is the Shannon entropy formula suitably normalized and applied to the power spectral density of the EEG signal (Sleigh et al 2004), while SampEn is an embedding entropy that quantifies the irregularity (or complexity) in data without the drawbacks that widely used nonlinear methods ( $D_{2}$ and L1) have (Richman and Moorman 2000). We wanted to test the 
hypothesis that the entropy of the AD patients' EEG would be different than that of agematched controls, hence indicating an abnormal type of dynamics in this group.

The paper is organized as follows. In Section 2 we explain the selection of patients and controls, the EEG recording and how were artefact-free epochs chosen. SampEn and SpecEn are also introduced in Section 2, as well as the statistical tools used to evaluate the differences between entropy values for AD patients and control subjects. Section 3 presents the results of our study. Finally, in Section 4 we discuss our results and compare them with other studies of the EEG background activity in AD patients with non-linear analysis methods, and we draw our conclusions.

\section{Materials and Methods}

\subsection{Subjects and signals}

Twenty-two subjects participated in this study. Eleven patients ( 5 men and 6 women; age $=72.5$ \pm 8.3 years, mean \pm standard deviation $\mathrm{SD}$ ) fulfilling the criteria of probable AD were recruited from the Alzheimer's Patients' Relatives Association of Valladolid (AFAVA) and referred to the University Hospital of Valladolid (Spain), where the EEG was recorded. All of them had undergone a thorough clinical evaluation that included clinical history, physical and neurological examinations, brain scans and a Mini-Mental State Examination (MMSE), generally accepted as a quick and simple way to evaluate cognitive function (Folstein et al 1975). The mean MMSE score for the patients was $13.1 \pm 5.9$ (Mean \pm SD), with five of them having a score of less than 12 points, indicating a severe degree of dementia. Two patients were receiving lorapezam. With therapeutic doses, benzodiapzepines may enhance beta activity, although no prominent rapid rhythms were observed in the visual examination of their EEGs. None of the other patients used medication that could be expected to influence the EEG.

The control group consisted of 11 age-matched, elderly control subjects without past or present neurological disorders ( 7 men and 4 women; age $=72.8 \pm 6.1$ years, mean \pm SD). The MMSE score value for all control subjects was 30 .

The local ethics committee approved the study. All control subjects and all caregivers of the demented patients gave their informed consent for participation in the current study.

\subsection{EEG recording}

EEGs were recorded from the 19 scalp loci of the international 10-20 system (electrodes Fp1, Fp2, F3, F4, C3, C4, P3, P4, O1, O2, F7, F8, T3, T4, T5, T6, Fz, Cz and Pz, all of them being referenced to the chin). More than five minutes of data were recorded from each subject using a Profile Study Room 2.3.411 EEG equipment (Oxford Instruments). Sample frequency was 256 $\mathrm{Hz}$, with a 12-bit A-to-D precision. Recordings were made with the subjects in a relaxed state and under the eyes-closed condition in order to obtain as many artefact-free EEG data as possible.

All EEGs were visually inspected by a specialist physician to check for eye movement and other artefacts. Thus, only EEG data free from electrooculographic and movement artefacts, and with minimal electromyographic (EMG) activity were selected for the entropy computations. Afterwards, EEGs were organized in 5 second artefact-free epochs (1280 points) that were copied as ASCII files for off-line analysis on a personal computer. An average number of $30.0 \pm 12.5$ artefact-free epochs (Mean $\pm \mathrm{SD}$ ) were selected from each electrode for each subject. Furthermore, all recordings were digitally filtered with a band-pass filter with cut-off frequencies at $0.5 \mathrm{~Hz}$ and at $40 \mathrm{~Hz}$ in order to remove EMG activity prior to the SampEn and SpecEn calculations.

\subsection{Spectral Entropy (SpecEn)}

Shannon defined the information concept of entropy as the expected value (i.e. the average amount) of the information of a probability distribution (Shannon 1948). Shannon's definition of entropy has been applied, modified and proven valid in a variety of fields. In 1979, Powell 
and Percival introduced Spectral Entropy (SpecEn), based on the peaks of the Fourier transform, as a measure of regularity (Powell and Percival 1979). This definition was also extended to the relative power spectral density of the EEG by Inouye et al (1991) and can be calculated with the following expression:

$$
H(f)=-\frac{1}{\ln (N)} \sum_{i=1}^{N} p_{i} \ln \left(p_{i}\right)
$$

where $p_{i}$ are the spectral amplitudes of frequency bin $i$ (assuming a bin width of one spectral unit), the sum of all $p_{i}$ is equal to 1 , and $N$ is the number of frequency bins (Sleigh et al 2004). The $p_{i}$ can be obtained as the normalized value of the power spectral density at each frequency bin (Rezek and Roberts 1998).

SpecEn is a convenient way of quantifying the distribution of spectral power, as it measures the flatness of frequency spectrum. A high SpecEn implies a flat, uniform spectrum with a broad spectral content, whereas a low SpecEn implies a spectrum with all the power condensed into a single frequency bin (i.e. a less complex, more predictable signal) (Sleigh et al 2004). This entropy estimator has been applied to measure the irregularity of the EEG during rest and mental arithmetic tasks, with results showing that EEGs during rest were significantly more irregular anteriorly than in the occipital areas (Inouye et al 1991). Fell et al (1996) have shown that SpecEn is a useful discriminator of sleep stages, as its value decreases significantly from stage II to stages III and IV. Moreover, the use of SpecEn to study epilepsy shows promise. The background EEG is disorganized in or near the epileptogenic focus and focal background abnormalities can therefore be estimated by SpecEn (Inouye et al 1992). It has been demonstrated that this entropy measure is useful in the extraction of features from EEG recordings of a patient during Cheyne-Stokes respiration (Rezek and Roberts 1998). Furthermore, SpecEn has been used to monitor the depth of anaesthesia (Rezek and Roberts 1998, Zhang and Roy 2001), and the algorithm has even been implemented in the DatexOhmeda S/5 ${ }^{\mathrm{TM}}$ Entropy module (Viertiö-Oja et al 2004). SpecEn can be used to detect subtle changes in the EEG background activity. It has been shown that it decreases abruptly as the patient becomes unconscious during induction of general anaesthesia, and does not decrease significantly with further deepening of the anaesthesia (Sleigh et al 2004).

In this pilot study we estimated the SpecEn of the EEG background activity of AD patients and control subjects with a short computer program, developed with MATLAB ${ }^{\circledR}$. The spectral power density was obtained as the Fourier transform of the autocorrelation function of the EEG signals. The different $p_{i}$ were obtained as the normalized value of the power spectral density, with respect to the total spectral power in the 0.5 to $40 \mathrm{~Hz}$ band, at each frequency. We wanted to test if this method could be useful for the detection of the differences in the power spectrum between both groups, as one of typical abnormalities in AD is a shift of the power spectrum to lower frequencies.

\subsection{Sample Entropy (SampEn)}

As we have previously mentioned, embedding entropies provide information about how the EEG signal fluctuates with time by comparing the time series with a delayed version of itself (Sleigh et al 2004). Several embedding-based formulas have been proposed in an attempt to estimate the Kolmogorov-Sinai (KS) entropy with reasonable precision, such as $K_{2}$ entropy (Grassberger and Procaccia 1983c) or the Eckmann and Ruelle entropy (Eckmann and Ruelle 1985). However, these methods to estimate the entropy of a system represented by a time series are usually not well suited to short and noisy data sets, such as biomedical signals. To overcome this drawback, Pincus introduced a family of statistics named Approximate Entropy (ApEn) (Pincus 1991). ApEn provides a widely applicable, statistically valid formula that will distinguish data sets by a measure of regularity (Pincus 1991).

$A p E n$ examines time series for similar epochs and assigns a non-negative number to the sequence, with larger values corresponding to more complexity or irregularity in the data (Pincus 1991). Briefly, given $N$ points, $\operatorname{ApEn}(m, r, N)$ measures the logarithmic likelihood that runs of patterns that are close (within $r$ ) for $m$ contiguous observations remain close (within the 
same tolerance width $r$ ) on subsequent incremental comparisons. The ApEn algorithm counts each sequence as matching itself to avoid the occurrence of $\ln (0)$ in the calculations and this has led to discussion of the bias of ApEn (Richman and Moorman 2000).

To reduce this bias, Richman and Moorman have developed and characterized a new family of statistics: SampEn (Richman and Moorman 2000). Two input parameters, a run length $m$ and a tolerance window $r$, must be specified to compute $\operatorname{SampEn}$. $\operatorname{SampEn}(m, r, N)$ is the negative logarithm of the conditional probability that two sequences similar for $m$ points remain similar at the next point, where self-matches are not included in calculating the probability. Thus, a lower value of SampEn also indicates more self-similarity in the time series. SampEn is largely independent of record length and displays relative consistency under circumstances where ApEn does not (Richman and Moorman 2000). In addition to eliminating self-matches, the SampEn algorithm is simpler than the ApEn algorithm, requiring one-half as much time to calculate.

Formally, given $N$ data points from a time series $\{x(n)\}=x(1), x(2), \ldots, x(N)$, to define SampEn, one should follow these steps:

1. Form $m$-vectors $X_{m}(1) \ldots X_{m}(N-m+1)$ defined by $X_{m}(i)=[x(i), x(i+1), \ldots, x(i+m-1)]$, for 1 $\leq i \leq N-m+1$. These vectors represent $m$ consecutive $x$ values, commencing with the $i$ th point.

2. Define the distance between vectors $X_{m}(i)$ and $X_{m}(j), d\left[X_{m}(i), X_{m}(j)\right]$, as the maximum absolute difference between their scalar components:

$$
d\left[X_{m}(i), X_{m}(j)\right]=\max _{k=0, \ldots, m-1}(|x(i+k)-x(j+k)|)
$$

3. For a given $X_{m}(i)$, count the number of $j(1 \leq j \leq N-m, j \neq i)$, denoted as $B_{i}$, such that the distance between $X_{m}(i)$ and $X_{m}(j)$ is less than or equal to $r$. Then, for $1 \leq i \leq N-m$,

$$
B_{i}^{m}(r)=\frac{1}{N-m-1} B_{i}
$$

4. Define $B^{m}(r)$ as:

$$
B^{m}(r)=\frac{1}{N-m} \sum_{i=1}^{N-m} B_{i}^{m}(r)
$$

5. We increase the dimension to $m+1$ and calculate $A_{i}$ as the number of $X_{m+1}(i)$ within $r$ of $X_{m+1}(j)$, where $j$ ranges from 1 to $N-m(j \neq i)$. We then define $A_{i}^{m}(r)$ as:

$$
A_{i}^{m}(r)=\frac{1}{N-m-1} A_{i}
$$

6. We set $A^{m}(r)$ as:

$$
A^{m}(r)=\frac{1}{N-m} \sum_{i=1}^{N-m} A_{i}^{m}(r)
$$

Thus, $B^{m}(r)$ is the probability that two sequences will match for $m$ points, whereas $A^{m}(r)$ is the probability that two sequences will match for $m+1$ points.

We define Sample Entropy by:

$$
\operatorname{SampEn}(m, r)=\lim _{N \rightarrow \infty}\left\{-\ln \left[\frac{A^{m}(r)}{B^{m}(r)}\right]\right\}
$$

which is estimated by the statistic:

$$
\operatorname{SampEn}(m, r, N)=-\ln \left[\frac{A^{m}(r)}{B^{m}(r)}\right]
$$

It is imperative to consider $\operatorname{SampEn}(m, r, N)$ as a family of parameters: comparisons are intended with fixed $m, r$ and $N . N$ is the length of the time series, $m$ is the length of the sequences to be compared and $r$ is the tolerance for accepting matches. It is convenient to set the 
tolerance as $r$ times the standard deviation of the original data sequence. This gives SampEn scale invariance, in that it remains unchanged under uniform process magnification, reduction, or constant shift to higher or lower values (Pincus 1991, Richman and Moorman 2000).

Although $m$ and $r$ are critical in determining the outcome of SampEn, no guidelines exist for optimizing their values. In principle, the accuracy and confidence of the entropy estimate improve as the number of matches of length $m$ and $m+1$ increases. The number of matches can be increased by choosing small $m$ (short templates) and large $r$ (wide tolerance) (Lake et al 2002). However, there are penalties for criteria that are too relaxed (Pincus 1991). For smaller $r$ values, one usually achieves poor conditional probability estimates, while for larger $r$ values, too much detailed system information is lost and SampEn tends to 0 for all processes.

Despite its advantages over $A p E n$, the use of SampEn is not widespread. It has been used to study abnormal heart rate (HR) characteristics of reduced variability in the early course of neonatal sepsis, where it has been shown that SampEn of neonatal HR falls before the clinical diagnosis of sepsis (Lake et al 2002). Moreover, this entropy measure has been applied to characterize the non-linear features of HR time series for three recumbent positions (Kim et al 2005). To our knowledge, this is the first study of the EEG background activity with SampEn.

Because of the non-linear character of EEG signals, SampEn can be used as a powerful tool in the study of the EEG background activity. For this study, SampEn was calculated with a short computer program, developed with MATLAB ${ }^{\circledR}$, and parameter values $m=1$ and $r=0.25$ times the standard deviation of the original data sequence. SampEn results for these set of values are in agreement with theoretical entropy estimates for short data sets as the EEG epochs we are analysing (Richman and Moorman 2000).

\subsection{Statistical analysis}

Student's $t$-test was used to evaluate the statistical differences between the estimated SpecEn and SampEn values for AD patients and control subjects. If the $p$ value was lower than 0.01 , the differences between the mean values were considered significant.

The ability of the entropy methods to discriminate $\mathrm{AD}$ patients from control subjects at the electrodes where $p<0.01$ was evaluated using Receiver Operating Characteristic (ROC) curves (Zweig and Campbell 1993). ROC curves can be obtained by plotting the sensitivity values (the proportion of patients with a diagnosis of $\mathrm{AD}$ who test positive, i.e. the true positive rate) on the $y$ axis against their equivalent $\{1$-specificity $\}$ values (specificity represents the percentage of controls correctly recognized, i.e. the true negative rate) for all the available cutoff points (in this case, the SpecEn or SampEn values) on the $x$ axis. We used a computer program developed with MATLAB ${ }^{\circledR}$ that automatically selected different cut-off points and calculated the sensitivity/specificity pair for each one of them. Accuracy is a related parameter that quantifies the total number of subjects (AD patients and control subjects) precisely classified. The optimum threshold is the cut-off point in which the highest accuracy (minimal false negative and false positive results) is obtained. It can be determined from the ROC curve as the closest value to the left top corner (100\% sensitivity, $100 \%$ specificity).

\section{Results}

SpecEn and $\operatorname{SampEn}(m=1, r=0.25$ times the standard deviation of the data) were estimated for channels Fp1, Fp2, F3, F4, C3, C4, P3, P4, O1, O2, F7, F8, T3, T4, T5 and T6. The results were averaged based on all the artefact-free 5 second epochs $(N=1280$ points $)$ within the five-minute period of EEG recordings.

The SpecEn values (Mean \pm SD) for the AD patients and control subjects and the $p$ values of the Student's t-tests performed to examine the differences between both groups are summarized in table 1. As it can be seen, although the control subjects' spectral entropy values were higher than those of the AD patients at most electrodes, no significant differences were found between both groups $(p>0.01)$.

Table 2 summarizes the SampEn values (Mean \pm SD) for the AD patients and control subjects and the $p$-values of the Student's $t$-test. AD patients had lower SampEn values than 
control subjects at all electrodes apart from T4, with significant differences between both groups at electrodes O1, O2, P3 and P4 $(p<0.01)$. These results suggest that EEG activity of AD patients is more regular (less complex) than in a normal brain in the parietal and occipital regions.

Table 1. Average SpecEn values of the EEGs for the AD patients and control subjects for all channels.

\begin{tabular}{cccc}
\hline Electrode & $\begin{array}{c}\text { Control subjects } \\
\text { (Mean } \pm \text { SD) }\end{array}$ & $\begin{array}{c}\text { AD patients } \\
\text { (Mean } \pm \text { SD })\end{array}$ & $\begin{array}{c}\text { Statistical } \\
\text { analysis }\end{array}$ \\
\cline { 4 - 4 } F3 & $0.6176 \pm 0.0721$ & $0.6207 \pm 0.1024$ & 0.9356 \\
F4 & $0.6906 \pm 0.0855$ & $0.6477 \pm 0.0938$ & 0.2755 \\
F7 & $0.6612 \pm 0.1153$ & $0.6758 \pm 0.1027$ & 0.7571 \\
F8 & $0.7035 \pm 0.1126$ & $0.6746 \pm 0.1064$ & 0.5437 \\
Fp1 & $0.6604 \pm 0.1083$ & $0.6302 \pm 0.1216$ & 0.5455 \\
Fp2 & $0.6556 \pm 0.1133$ & $0.6094 \pm 0.1011$ & 0.3241 \\
T3 & $0.7515 \pm 0.0845$ & $0.7102 \pm 0.1176$ & 0.3552 \\
T4 & $0.7821 \pm 0.0739$ & $0.7110 \pm 0.0926$ & 0.0605 \\
T5 & $0.7073 \pm 0.0696$ & $0.6726 \pm 0.0735$ & 0.2691 \\
T6 & $0.7572 \pm 0.1030$ & $0.6629 \pm 0.1244$ & 0.0671 \\
C3 & $0.6634 \pm 0.1112$ & $0.6273 \pm 0.1143$ & 0.4609 \\
C4 & $0.6749 \pm 0.1143$ & $0.6165 \pm 0.1281$ & 0.2730 \\
P3 & $0.7129 \pm 0.0814$ & $0.6383 \pm 0.1144$ & 0.0933 \\
P4 & $0.7231 \pm 0.0980$ & $0.6416 \pm 0.1027$ & 0.0713 \\
O1 & $0.7320 \pm 0.0861$ & $0.6822 \pm 0.0802$ & 0.1759 \\
O2 & $0.7413 \pm 0.0978$ & $0.6769 \pm 0.0729$ & 0.0953 \\
\hline
\end{tabular}

Table 2. Average $\operatorname{SampEn}(1,0.25,1280)$ values of the EEGs for the AD patients and control subjects for all channels. Significant group differences are marked with an asterisk.

\begin{tabular}{cccc}
\hline Electrode & $\begin{array}{c}\text { Control subjects } \\
(\text { Mean } \pm \text { SD) }\end{array}$ & $\begin{array}{c}\text { AD patients } \\
(\text { Mean } \pm \text { SD })\end{array}$ & $\begin{array}{c}\text { Statistical } \\
\text { analysis }\end{array}$ \\
\cline { 4 - 4 } & $0.6551 \pm 0.1867$ & $0.5759 \pm 0.1048$ & 0.2342 \\
F3 & $0.6473 \pm 0.1796$ & $0.6357 \pm 0.1237$ & 0.8610 \\
F4 & $0.7030 \pm 0.1859$ & $0.6694 \pm 0.1450$ & 0.6425 \\
F7 & $0.7162 \pm 0.1645$ & $0.6636 \pm 0.1425$ & 0.4325 \\
F8 & $0.6533 \pm 0.1471$ & $0.5177 \pm 0.1803$ & 0.0677 \\
Fp1 & $0.6371 \pm 0.1966$ & $0.5279 \pm 0.1196$ & 0.1316 \\
Fp2 & $0.8783 \pm 0.2697$ & $0.8481 \pm 0.2275$ & 0.7798 \\
T3 & $0.8513 \pm 0.2254$ & $0.8629 \pm 0.3003$ & 0.9189 \\
T4 & $0.8284 \pm 0.1835$ & $0.6329 \pm 0.1867$ & 0.0223 \\
T5 & $0.8141 \pm 0.1908$ & $0.6327 \pm 0.1972$ & 0.0404 \\
T6 & $0.7578 \pm 0.1484$ & $0.6652 \pm 0.1794$ & 0.2020 \\
C3 & $0.7670 \pm 0.1178$ & $0.7040 \pm 0.1987$ & 0.3765 \\
C4 & $0.7781 \pm 0.1201$ & $0.5576 \pm 0.1625$ & 0.0017 \\
P3* & $0.7852 \pm 0.1192$ & $0.5859 \pm 0.1547$ & 0.0029 \\
P4* & $0.8849 \pm 0.1672$ & $0.6361 \pm 0.1745$ & 0.0027 \\
O1* & $0.8538 \pm 0.1899$ & $0.6278 \pm 0.1756$ & 0.0089 \\
O2* & & &
\end{tabular}

Finally, we evaluated the ability of SampEn to discriminate AD patients from control subjects at the electrodes in which significant differences were found using ROC plots (Zweig and Campbell 1993). The highest sensitivity was obtained at electrode O2 (90.91\%), although with a small specificity $(63.64 \%)$. On the other hand, the highest specificity was reached at electrode P4 (90.91\%), with a decreased sensitivity (63.64\%). The accuracy of the diagnostic test was similar at all four electrodes in which significant differences between both groups were 
found $(77.27 \%)$. However, sensitivity was higher than specificity on the occipital electrodes, while specificity was higher than sensitivity on the parietal channels. Table 3 summarizes these results.

Table 3. Test results for $\operatorname{SampEn}(1,0.25,1280)$ on the channels in which the differences between both groups were significant. The optimum threshold to discriminate AD patients and control subjects is included.

\begin{tabular}{cccccc}
\hline Electrode & Threshold & $\begin{array}{c}\text { Sensitivity } \\
(\%)\end{array}$ & $\begin{array}{c}\text { Specificity } \\
(\%)\end{array}$ & $\begin{array}{c}\text { Accuracy } \\
(\%)\end{array}$ & $\begin{array}{c}\text { Area under } \\
\text { the ROC } \\
\text { curve }\end{array}$ \\
\hline P3 & 0.6658 & 72.73 & 81.82 & 77.27 & 0.8512 \\
P4 & 0.6740 & 63.64 & 90.91 & 77.27 & 0.8347 \\
O1 & 0.7492 & 81.82 & 72.73 & 77.27 & 0.8595 \\
O2 & 0.7367 & 90.91 & 63.64 & 77.27 & 0.7769 \\
\hline
\end{tabular}

The value for the area under the ROC curve can be interpreted as follows: an area of 0.8595 (electrode $\mathrm{O} 1$, for example) means that a randomly selected individual from the control subjects' group has a SampEn value larger than that of a randomly chosen individual from the AD patients' group in $85.95 \%$ of the time (Zweig and Campbell 1993). A rough guide to classify the precision of a diagnostic test is related to the area under the ROC curve. With values between 0.90 and 1 the precision of the diagnostic test is considered to be excellent, good for values between 0.80 and 0.89 , fair if the results are in the range $0.70-0.79$, poor when the value of the area under the ROC curve is between 0.60 and 0.69 , and bad for values between 0.50 and 0.59 . Thus, the results obtained can be considered good for electrodes P3, P4 and O1, and fair for electrode O2. Figure 1 depicts the ROC curves corresponding to the SampEn analysis.

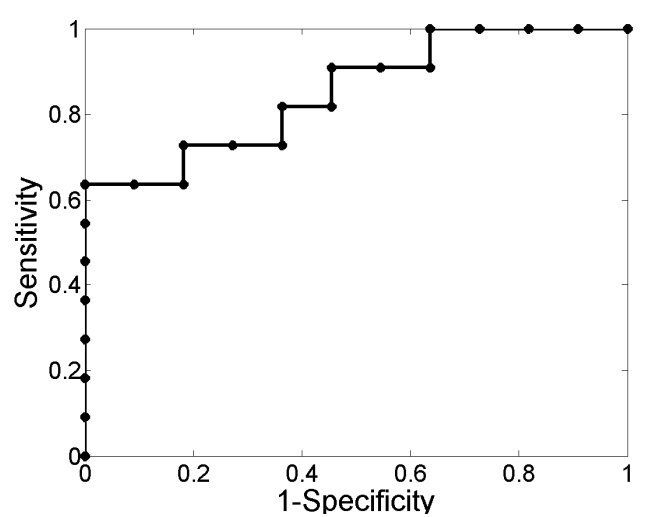

(a)

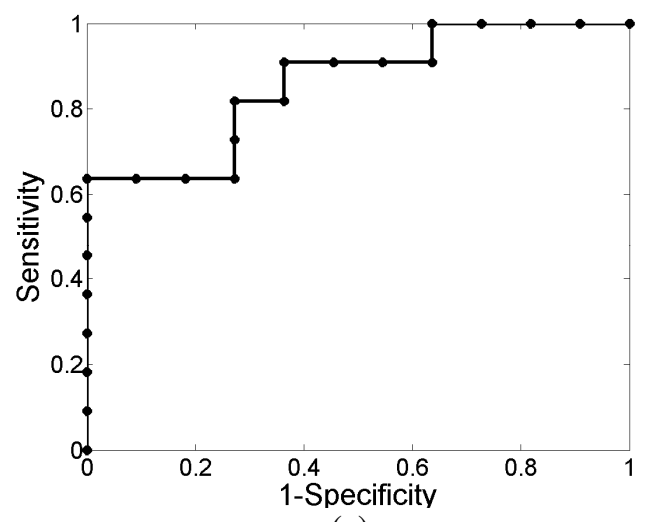

(c)

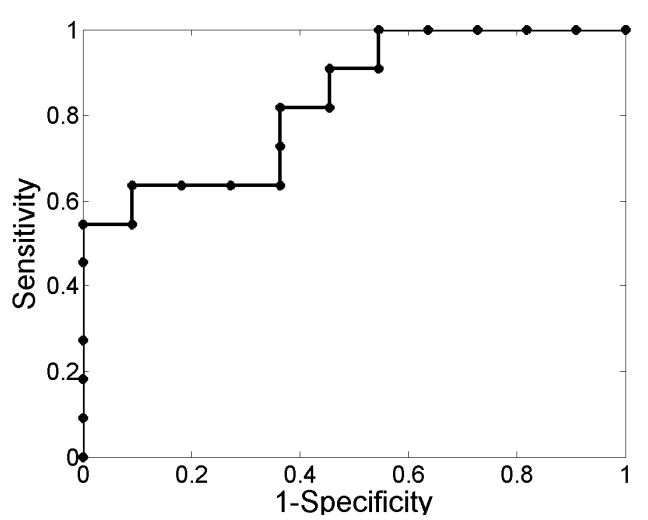

(b)

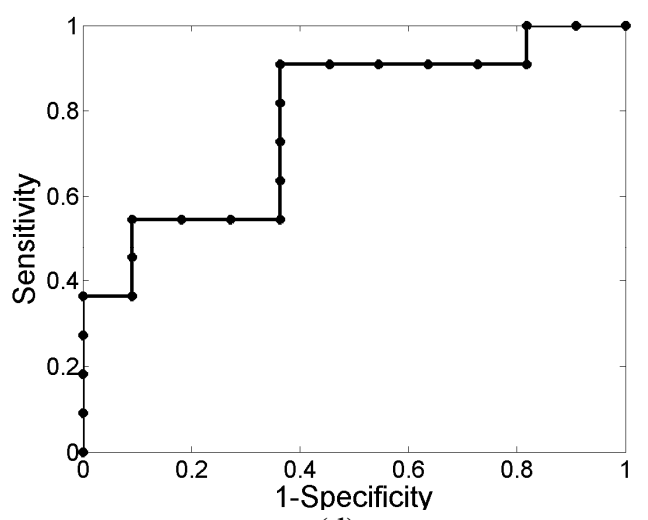

(d)

Figure 1. ROC curves for the SampEn values at the electrodes in which $p<0.01$. (a) P3. (b) P4. (c) O1. (d) O2. 


\section{Discussion and Conclusions}

In this pilot study we have analysed the EEG background activity of 11 control subjects and 11 patients with AD applying SampEn and SpecEn. SampEn is an embedding entropy related to ApEn. SampEn statistics provide an improved evaluation of time series irregularity, with increasing values corresponding to intuitively increasing process complexity. SpecEn is the Shannon entropy formula suitably normalized and applied to the power spectral density of the signal, and is a convenient way of quantifying the distribution of spectral power.

SpecEn has proven not to be effective in discriminating AD patients from control subjects. Although the mean SpecEn values were higher at most electrodes for control subjects than for AD patients, the differences were not significant $(p>0.01)$. One of the most relevant EEG abnormalities in AD patients is a shift of the power spectrum to lower frequencies, through increase of the delta $(0.5-4 \mathrm{~Hz})$ and theta $(4-8 \mathrm{~Hz})$ power, along with decrease of the alpha $(8-$ $13 \mathrm{~Hz}$ ) power (Jeong 2004). SpecEn quantifies the distribution of spectral power in the EEG. However, our results show that, contrary to what we expected, SpecEn is unable to reflect the power spectrum changes usually found in AD. This might be due to the fact that the spectral distribution of power in the EEG of $\mathrm{AD}$ patients is shifted to lower frequencies, but the shape of the spectrum remains relatively unchanged. Despite these negative results, the possible usefulness of SpecEn in the diagnosis of AD should be investigated with a larger number of patients and control subjects.

On the other hand, we have found that AD patients have significantly lower SampEn values than control subjects at electrodes $\mathrm{O} 1, \mathrm{O} 2, \mathrm{P} 3$ and $\mathrm{P} 4(p<0.01)$. We infer that brains affected by $\mathrm{AD}$ show a more regular and less complex electrophysiological behaviour in the parietal and occipital regions. This confirms findings associated with the fact that a diffuse slowing of the background activity may be found in the EEG of patients with AD (Markand 1990). Moreover, our results agree with other studies showing that the EEG of AD patients has lower $D_{2}$ values than that of control subjects (Pritchard et al 1994, Stam et al 1995, Jeong et al 1998, 2001a, Jelles et al 1999) and, consequently, a less complex brain activity. Besthorn et al (1995) found that a lower $D_{2}$ was correlated with increased severity of dementia and that this method correctly classified AD patients and controls with an accuracy of 70\% (Besthorn et al 1997). Pritchard et al (1994) showed that the addition of non-linear measures $\left(D_{2}\right)$ and a neural net classification procedure to linear methods improves the classification accuracy of the $\mathrm{AD} /$ control status of subjects up to $92 \%$. Jeong et al $(1998,2001 \mathrm{a})$ found that AD patients have significantly lower $L 1$ values than age-matched controls. The $L 1$ of the EEG can be interpreted as a measure of flexibility of information processing in the brain (Röschke et al 1995). In this context, decreased $L 1$ values in $\mathrm{AD}$ patients reflect a drop in the flexibility of information processing in the injured brain (Jeong 2004). The decreased complexity of brain activity in AD patients has also been shown using Lempel-Ziv complexity (Abásolo et al 2005b), with an accuracy of $81.82 \%$ at some electrodes. Furthermore, the aforementioned increased regularity in the EEG background activity in AD patients has also been found in the parietal electrodes with ApEn (Abásolo et al 2005a). The results of this study suggest that ApEn might be complementary to spectral and autocorrelation analyses.

The reduction of irregularity in the EEG of AD patients could be explained by a decrease of dynamical complexity of part of the brain. However, the pathophysiological implications of this decreased EEG irregularity (or complexity) are not clear. Among others, three mechanisms can be responsible for it: neuronal death, a general effect of neurotransmitter deficiency and loss of connectivity of local neural networks as a result of nerve cell death (Jelles et al 1999, Jeong 2004). Nevertheless, ageing and age-related diseases often accompany a wide-ranging loss of physiological complexity (Kyriazis, 2003). As the AD patients' and control subjects' groups were carefully matched for age, the increase of regularity in the parietal and occipital regions might represent the cognitive dysfunction in AD. However, a possible association should be investigated with a larger number of patients and control subjects.

In order to estimate SampEn accurately we need recordings of just $10^{m}$ to $20^{m}$ points, where $m$ is the run length that must be fixed to compute this embedding entropy. Thus, this 
family of statistics is much better suited for EEG analysis than traditional non-linear techniques like $L 1$ or $D_{2}$, that require an amount of data to obtain meaningful results usually beyond the experimental possibilities for physiological data (Eckmann and Ruelle 1992). Moreover, SampEn statistics (Richman and Moorman 2000):

- agree much better than $A p E n$ with theory for random numbers with known probabilistic character over a broad range of operating conditions,

- maintain relative consistency where ApEn statistics do not, and

- have residual bias for very short record lengths.

These properties make SampEn an attractive tool for non-linear analysis of biomedical signals.

Some limitations of our study merit consideration. First of all, although AD patients had lower SampEn values than control subjects at all electrodes apart from T4, only four electrodes ( $\mathrm{P} 3, \mathrm{P} 4, \mathrm{O} 1$ and $\mathrm{O} 2)$ showed statistically significant differences between both groups. The symmetric disposition of those electrodes over the scalp shows promise and might reflect significant changes in the brain electrical activity of $\mathrm{AD}$ patients in the parietal and occipital regions. However, caution should be applied due to the preliminary nature of this study. Furthermore, the sample size was small. Hence, to prove the usefulness of SampEn as a diagnostic tool, this approach should be extended on a much larger patient population. Moreover, the detected increase of EEG regularity (or decrease of complexity) is not specific to AD. It appears in several physiological and pathological states including, among others, sleep (Burioka et al 2003), anaesthesia (Zhang and Roy 2001), the Creutzfeld-Jakob disease (Babloyantz and Destexhe 1988), vascular dementia (Jeong et al 1998), schizophrenia (Röschke et al 1995) and Parkinson's disease (Stam et al 1995). Thus, although this pilot study shows that SampEn might be a helpful tool for recognition of $\mathrm{AD}$, further work must be carried out to examine non-linear EEG activity in other types of dementia.

In summary, although non-linear EEG analysis cannot yet be applied as a diagnostic tool, our findings show the possibility to analyse the dynamical behaviour of the brain in AD patients and to detect significant differences with SampEn. Our experimental results prove the potential applications of this new family of statistics in reflecting differences in the irregularity of EEG data time series of patients with a diagnosis of AD and control subjects. Non-linear dynamics suggest that $\mathrm{AD}$ can be a dynamical disease which is characterized by changes in the qualitative dynamics of physiological processes (Belair et al 1995). The decrease of the EEG entropy found in the parietal and occipital regions in AD patients leads us to think that EEG analysis with SampEn could be a useful tool to increase our insight into brain dysfunction in this disease.

\section{Acknowledgments}

This work has been partially supported by the grant project from Consejería de Sanidad de la Junta de Castilla y León VA15/04. We are very grateful to María Luisa Baz, Ph.D., who kindly revised the English version of this paper.

\section{References}

Abásolo D, Hornero R, Espino P, Poza J, Sánchez C I and de la Rosa R 2005a Analysis of regularity in the EEG background activity of Alzheimer's disease patients with Approximate Entropy Clin. Neurophysiol. 116 1826-34

Abásolo D, Hornero R, Espino P, Poza J, Sánchez C I and de la Rosa R 2005b Analysis of EEG background activity in Alzheimer's disease patients with Lempel-Ziv complexity and Central Tendency Measure Med. Eng. Phys. In press.

Babloyantz A and Destexhe A 1988 The Creutzfeldt-Jakob disease in the hierarchy of chaotic attractors From chemical to biological organization ed M Markus, S Müller et al (Berlin: Springer-Verlag) pp 307-16

Belair J, Glass L, an der Heiden U and Milton J 1995 Dynamical disease: identification, temporal aspects and treatment strategies of human illness Chaos 5 1-7 
Besthorn C, Sattel H, Geiger-Kabisch C, Zerfass R and Förstl H 1995 Parameters of EEG dimensional complexity in Alzheimer's disease Electroenceph. Clin. Neurophysiol. 95 84-89

Besthorn C, Zerfass R, Geiger-Kabisch C, Sattel H, Daniel S, Schreiter-Gasser U and Förstl H 1997 Discrimination of Alzheimer's disease and normal aging by EEG data Electroenceph. Clin. Neurophysiol. 103 241-8

Bird T D 2001 Alzheimer's disease and other primary dementias Harrison's Principles of Internal Medicine ed E Braunwald, A S Fauci et al (New York: The McGraw-Hill Companies Inc) pp 2391-9

Burioka N, Cornélissen G, Halberg F, Kaplan D T, Suyama H, Sako T and Shimizu E 2003 Approximate entropy of human respiratory movement during eye-closed waking and different sleep stages Chest $12380-6$

Eckmann J P and Ruelle D 1985 Ergodic theory of chaos and strange attractors Rev. Mod. Physics 57 $617-56$

Eckmann J P and Ruelle D 1992 Fundamental limitations for estimating dimensions and Lyapunov exponents in dynamical systems Physica D 56 185-7

Fell J, Röschke J, Mann K and Schäffner C 1996 Discrimination of sleep stages: a comparison between spectral and nonlinear EEG measures Electroenceph. Clin. Neurophysiol. 98 401-10

Folstein M F, Folstein S E and McHugh P R 1975 Mini-mental state. A practical method for grading the cognitive state of patients for the clinician J. Psychiatr. Res. 12 189-98

Grassberger P and Procaccia I 1983a Measuring the strangeness of strange attractors Physica D 9 189208

Grassberger P and Procaccia I 1983b Characterization of strange attractors Phys. Rev. Lett. 50 346-9

Grassberger P and Procaccia I 1983c Estimation of the Kolmogorov entropy of a chaotic signal Phys. Rev. A 28 2591-3

Inouye T, Shinosaki K, Sakamoto H, Toi S, Ukai S, Iyama A, Katsuda Y and Hirano M 1991 Quantification of EEG irregularity by use of the entropy of the power spectrum Electroenceph. Clin. Neurophysiol. 79 204-10

Inouye T, Shinosaki K, Sakamoto H, Toi S, Ukai S, Iyama A, Katsuda Y and Hirano M 1992 Abnormality of background EEG determined by the entropy of power spectra in epileptic patients Electroenceph. Clin. Neurophysiol. 82 203-7

Jelles B, van Birgelen J H, Slaets J P J, Hekster R E M, Jonkman E J and Stam C J 1999 Decrease of nonlinear structure in the EEG of Alzheimer patients compared to healthy controls Clin. Neurophysiol. $1101159-67$

Jeong J, Kim S J and Han S H 1998 Non-linear dynamical analysis of the EEG in Alzheimer's disease with optimal embedding dimension Electroenceph. Clin. Neurophysiol. 106 220-8

Jeong J, Chae J H, Kim S Y and Han S H 2001a Nonlinear dynamic analysis of the EEG in patients with Alzheimer's disease and vascular dementia J. Clin. Neurophysiol. 18 58-67

Jeong J, Gore J C and Peterson B S 2001b Mutual information analysis of the EEG in patients with Alzheimer's disease Clin. Neurophysiol. 112 827-35

Jeong J 2004 EEG dynamics in patients with Alzheimer's disease Clin. Neurophysiol. 115 1490-505

Kantz H and Schreiber T 1997 Nonlinear Time Series Analysis (Cambridge, England: Cambridge University Press)

Kim W-S, Yoon Y-Z, Bae J-H and Soh K-S 2005 Nonlinear characteristics of heart rate time series: influence of three recumbent positions in patients with mild or severe coronary artery disease Physiol. Meas. 26 517-29

Kyriazis M 2003 Practical applications of chaos theory to the modulation of human ageing: nature prefers chaos to regularity Biogerontology 4 75-90

Lake D E, Richman J S, Griffin M P and Moorman J R 2002 Sample entropy analysis of neonatal heart rate variability Am. J. Physiol. (Regul. Integr. Comp. Physiol.) 283 789-97

Markand O N 1990 Organic brain syndromes and dementias Current Practice of Clinical Electroencephalography ed D D Daly and T A Pedley (New York: Raven Press) pp 401-23

Pijnenburg Y A L, vd Made Y, van Cappellen van Walsum A M, Knol D L, Scheltens P and Stam C J 2004 EEG synchronization likelihood in mild cognitive impairment and Alzheimer's disease during a working memory task Clin. Neurophysiol. 115 1332-9

Pincus S M 1991 Approximate entropy as a measure of system complexity Proc. Natl. Acad. Sci. USA $\mathbf{8 8}$ 2297-2301

Powell G E and Percival I C 1979 A spectral entropy method for distinguishing regular and irregular motion of Hamiltonian systems J. Phys. A: Mat. Gen. 12 2053-71 
Pritchard W S, Duke D W, Coburn K L, Moore N C, Tucker K A, Jann M W and Hostetler R M 1994 EEG-based neural-net predictive classification of Alzheimer's disease versus control subjects is augmented by non-linear EEG measures Electroenceph. Clin. Neurophysiol. 91 118-30

Rezek I A and Roberts S J 1998 Stochastic complexity measures for physiological signal analysis IEEE Trans. Biomed. Eng. 45 1186-91

Richman J S and Moorman J R 2000 Physiological time-series analysis using approximate entropy and sample entropy Am. J. Physiol. (Heart Circ. Physiol.) 274 2039-49

Röschke J, Fell J and Beckmann P 1995 Non-linear analysis of sleep EEG data in schizophrenia: calculation of the principal Lyapunov exponent Psychiatr. Res. 56 257-69

Selkoe D J 1994 Cell biology of the amyloid beta-protein precursor and the mechanism of Alzheimer's disease, Annu. Rev. Cell. Biol. $10373-403$

Shannon C E 1948 A mathematical theory of communication Bell Syst. Tech. J. 27 379-423 623-56

Sleigh J W, Steyn-Ross D A, Grant C and Ludbrook G 2004 Cortical entropy changes with general anaesthesia: theory and experiment Physiol. Meas. 25 921-34

Stam C J, Jelles B, Achtereekte H A M, Rombouts S A R B, Slaets J P J and Keunen R W M 1995 Investigation of EEG nonlinearity in dementia and Parkinson's disease Electroenceph. clin. Neurophysiol. 95 309-17

Stam C J, van der Made Y, Pijnenburg Y A and Scheltens P 2003 EEG synchronization in mild cognitive impairment and Alzheimer's disease Acta Neurol. Scand. 108 90-6

Viertiö-Oja H, Maja V, Särkela M, Talja P, Tenkanen N, Tolvanen-Laakso H, Paloheimo M, Vakkuri A, Yli-Hankala A and Meriläinen P 2004 Description of the Entropy ${ }^{\mathrm{TM}}$ algorithm as applied in the Datex-Ohmeda S/5TM entropy module Acta Anesthesiol. Scand. 48 154-61

Wolf A, Swift J B, Swinney H L and Vastano J A 1985 Determining Lyapunov exponents from a timeseries Physica D 16 285-317

Zhang X S and Roy R J 2001 Derived fuzzy knowledge model for estimating the depth of anesthesia IEEE Trans. Biomed. Eng. 48 312-23

Zweig MH and Campbell G 1993 Receiver-Operating Characteristic (ROC) plots: a fundamental evaluation tool in clinical medicine Clin. Chem. 39 561-77 